\title{
SMOOTHING SPLINE IN A CONVEX CLOSED SET OF HILBERT SPACE
}

\section{Natasha Dicheva}

A characterisation of a smoothing spline is sought in a convex closed set $C$ of Hilbert space: $\min \left\{\alpha\|T x\|_{Y}^{2}+\|A x-z\|_{Z}^{2}, x \in C\right\}, T$ and $A$ are linear operators. A representation of the solution is obtained in the terms of the kernels of the above operators, of the dual operators $T^{*}, A^{*}$ and of the dual cone $C^{0}$. A particular case is considered when $T$ is the differential operator and $A$ is the operator-trace of a function.

Let $X, Y, Z$ be Hilbert spaces with scalar products respectively $(,)_{X},(,)_{Y},(,)_{Z}$. We are given linear bounded operators

$$
A: X \rightarrow Z, \quad T: X \rightarrow Y .
$$

Consider the operator equation $A x=z_{0}, z_{0} \in Z$.

1. If $A^{-1}\left(z_{0}\right) \neq \emptyset$, then $\sigma \in X$ is called an interpolating spline, if the following minimum is reached

$$
\|T \sigma\|_{Y}^{2}=\min _{x \in A^{-1}\left(z_{0}\right)}\|T x\|_{Y}^{2}
$$

2. If $A^{-1}\left(z_{0}\right)=\emptyset$, we introduce a real parameter $\alpha>0$ and construct a quadratic functional

$$
\phi_{\alpha}(x)=\alpha\|T x\|_{Y}^{2}+\left\|A x-z_{0}\right\|_{Z}^{2}
$$

We say that $\sigma_{*} \in X$ is a smoothing spline, if

$$
\phi_{\alpha}\left(\sigma_{*}\right)=\min _{x \in X} \phi_{\alpha}(x)
$$

Characterisations of the solutions of problems (1) and (3) are given in [5].

A certain shape of the interpolating or smoothing spline is required in many applied problems. The characterisation of such conditions can be often described by a set $C \subset X$, which is convex and closed.

\section{Received 21st November, 2001}

Copyright Clearance Centre, Inc. Serial-fee code: 0004-9727/02 \$A2.00+0.00. 
Chui, Deutsch and Ward give a characterisation of the solution of the problem for an interpolating spline in a convex set of Hilbert space $([3,4])$

$$
\min _{A x=z_{0}, x \in C}\|x\|^{2}
$$

In [2] a particular case of monotonicity is considered for both interpolating and smoothing splines. The characterisation is done from the point of view of a general optimisation problem in the terms of the Frechet-derivative and the polar cone.

We shall consider here the problem of finding a smoothing spline in a convex closed set of Hilbert space. That is, $\sigma_{*}$ is sought so that

$$
\phi_{\alpha}\left(\sigma_{*}\right)=\min _{x \in C \subset X} \phi_{\alpha}(x)
$$

where $\phi_{\alpha}(x)$ is the functional in (2). This problem arises for example, if the data are corrupted by noise and one does not require exact interpolation, but a special form of spline is required.

A new linear operator $L$ can be defined ([5]), which is acting on $F=Y \times Z$. If $f_{1}=\left[y_{1}, z_{1}\right], y_{1} \in Y, z_{1} \in Z, f_{2}=\left[y_{2}, z_{2}\right], y_{2} \in Y, z_{2} \in Z$, we define a scalar product in $F$ by

$$
\left(f_{1}, f_{2}\right)_{F}=\left(\left[y_{1}, z_{1}\right],\left[y_{2}, z_{2}\right]\right)_{F}:=\alpha\left(y_{1}, y_{2}\right)_{Y}+\left(z_{1}, z_{2}\right)_{Z}
$$

Let $L$ be the linear bounded operator

$$
L: X \rightarrow F, L x=[T x, A x]
$$

and let $a=\left[0_{Y}, z_{0}\right]$ be an element of $F$.

LEMMA 1. $\phi_{\alpha}(x)=\|L x-a\|_{F}^{2}$, where $a=\left[0_{Y}, z_{0}\right]$.

ProOF: By the definitions

$$
\begin{aligned}
& (L x-a, L x-a)_{F}=\left([T x, A x]-\left[0_{Y}, z_{0}\right],[T x, A x]-\left[0_{Y}, z_{0}\right]\right)_{F} \\
& =\left(\left[T x, A x-z_{0}\right],\left[T x, A x-z_{0}\right]\right)_{F}=\alpha(T x, T x)_{Y}+\left(A x-z_{0}, A x-z_{0}\right)_{Z} \\
& =\alpha\|T x\|^{2}+\left\|A x-z_{0}\right\|^{2}=\phi_{\alpha}(x) .
\end{aligned}
$$

Therefore $\phi_{\alpha}(x)=(L x-a, L x-a)_{F}=\|L x-a\|_{F}^{2}$.

Then the problem (5) is equivalent to

$$
\min _{f \in K}\|f-a\|^{2},
$$

where $K=L(C)=\{[y, z] \in Y \times Z: y=T x, z=A x, x \in C\}$.

Denote the kernels of $T$ and $A$ respectively by

$$
\text { ker } T=\left\{x \in X: T x=0_{Y}\right\}, \operatorname{ker} A=\left\{x \in X: A x=0_{Z}\right\} \text {. }
$$


LEMMA 2. If $T$ and $A$ are linear bounded operators and $\operatorname{ker} T \cap \operatorname{ker} A=\left\{0_{X}\right\}$, then $L$ is a linear bounded continuous operator and $\operatorname{ker} L=\left\{0_{X}.\right\}$

Proof: $L$ is a linear bounded operator, obviously. It follows it is continuous.

Let us show that $\operatorname{ker} L=\left\{0_{X}\right\}$. If $x \in \operatorname{ker} L$, that is, $L x=0_{F}$, then $L x=[T x, A x]$ $=\left[0_{Y}, 0_{Z}\right]$, therefore $T x=0_{Y}, A x=0_{Z}$, and $x \in \operatorname{ker} T \cap \operatorname{ker} A=0_{X}$, or $\operatorname{ker} L=0_{X}$.

The following lemma follows from the inverse operator theorem.

LEMmA 3. If $T$ and $A$ are linear bounded operators, $\operatorname{ker} T \cap \operatorname{ker} A=0_{X}$, and $L(X)$ is closed, then there exists $L^{-1}: L(X) \subset F \rightarrow X$ and $L^{-1}$ is a linear bounded continuous operator, too.

Here $L(X)=\{[y, z]: y=T x, z=A x, x \in X\}$.

We shall find conditions for closeness of $L(X)$ to be closed.

Lemma 4. $L(X)$ is closed if and only if $\operatorname{ker} T+\operatorname{ker} A$ is closed.

Proof: $L(X)$ is closed if and only if $L^{*}(F)=T^{*}(Y)+A^{*}(Z)$ is closed in $X$, if and only if $\operatorname{ker} T^{\perp}+\operatorname{ker} A^{\perp}$ is closed if and only if $\operatorname{ker} T+\operatorname{ker} A$ is closed.

Lemma 5. If $C$ is a closed convex subset of $X$, $\operatorname{ker} T \cap \operatorname{ker} A=\left\{0_{X}\right\}$, and $\operatorname{ker} T$ $+\operatorname{ker} A$ is closed, then $K=L(C)$ is a closed and convex subset of $F$.

Proof: Let $f_{1}, f_{2} \in L(C), \lambda_{1} \geqslant 0, \lambda_{2} \geqslant 0, \lambda_{1}+\lambda_{2}=1$. We shall show that $\lambda_{1} f_{1}+\lambda_{2} f_{2} \in L(C)$,too. There exists unique $x_{1} \in C: f_{1}=L x_{1}$, and $x_{2} \in C: f_{2}=L x_{2}$. We have $\lambda_{1} f_{1}+\lambda_{2} f_{2}=\lambda_{1} L x_{1}+\lambda_{2} L x_{2}=L\left(\lambda_{1} x_{1}+\lambda_{2} x_{2}\right) \in L(C)$, because $\lambda_{1} x_{1}+\lambda_{2} x_{2} \in C$.

Let's show, that $L(C)$ is closed. If $\left\{f_{n}\right\} \rightarrow f, f_{n} \in L(C)$, we shall show that $f$ $\in L(C)$.

There exists unique $x_{n} \in C: f_{n}=L x_{n}, L x_{n} \rightarrow f$. Applying the inverse continuous operator $L^{-1}$, it follows $L^{-1} L x_{n} \rightarrow L^{-1} f=x$.

So we have $x_{n} \rightarrow x$, but $C$ is closed, therefore $x \in C$. It means $f=L x \in L(C)$. $\quad$

THEOREM 1. If $C$ is a convex closed subset of $X, \operatorname{ker} T \cap \operatorname{ker} A=0_{X}$ and $\operatorname{ker} T$ $+\operatorname{ker} A$ is closed, then the problem (5) has the unique solution

$$
\sigma_{*}=L^{-1} P_{L(C)}(a)
$$

where $P_{L(C)}(a)$ denotes the orthogonal projection of $a$ on $L(C)$.

Proof: A classical result ([5, Theorem 2.1.2]) shows, that there exist unique solution of the problem (6) $f_{*} \in K$, such that

$$
\left\|f_{*}-a\right\|^{2}=\min _{f \in K}\|f-a\|^{2} .
$$

The point $f_{*} \in L(C)$, in which min $\|f-a\|$ is reached, is the orthogonal projection of $a$ on $L(C)$, that is, $f_{*}=P_{L(C)}(a)$. But $f_{*}=L \sigma_{*}$ for some $\sigma_{*}$, and $L$ is converse, (in according with Lemma 3 and 4), therefore the solution has the form

$$
\sigma_{*}=L^{-1}\left(P_{L(C)}(a)\right)
$$


Further we shall omit the brackets in $L(C)$ and denote $L C:=L(C)$. Define the dual operator $A^{*}$ of $A$ by

$$
(z, A x)=\left(A^{*} z, x\right)
$$

for all $z \in Z, x \in X$.

We denote the dual cone of $C$ by

$$
C^{0}=\{x \in X:(x, y) \leqslant 0, \forall y \in C\} .
$$

It is easy to see, that

$$
f_{*}=P_{L C}(a) \text { if and only if } a-f_{*} \in\left(L C-f_{*}\right)^{0}=(L C)^{0} \cap f_{*}^{\perp} .
$$

Theorem 2. Problem (5) has the unique solution $\sigma_{*}$ if and only if

$$
-\alpha T^{*} T \sigma_{*}+A^{*}\left(z_{0}-A \sigma_{*}\right) \in C^{0}
$$

and

$$
\Phi_{\alpha}\left(\sigma_{*}\right)=\left(z_{0}-A \sigma_{*}, z_{0}\right)
$$

ProOF: By (8), $a-f_{*} \in(L C)^{0}$ means, that

$$
\left(a-f_{*}, L \sigma\right) \leqslant 0, \forall \sigma \in C .
$$

But $f_{*} \in L(C)$, therefore there exist unique $\sigma_{*}$ such that $f_{*}=L \sigma_{*}=\left[T \sigma_{*}, A \sigma_{*}\right]$. Then

$$
\begin{gathered}
\left(a-f_{*}, L \sigma\right)=\left(\left[0 Y, z_{0}\right]-\left[T \sigma_{*}, A \sigma_{*}\right],[T \sigma, A \sigma]\right)=\left(\left[-T \sigma_{*}, z_{0}-A \sigma_{*}\right],[T \sigma, A \sigma]\right) \\
=-\alpha\left(T \sigma_{*}, T \sigma\right)+\left(z_{0}-A \sigma_{*}, A \sigma\right) \leqslant 0 .
\end{gathered}
$$

Therefore

$$
\left(-\alpha T^{*} T \sigma_{*}, \sigma\right)+\left(A^{*}\left(z_{0}-A \sigma_{*}\right), \sigma\right) \leqslant 0, \sigma \in C
$$

This means, that

$$
-\alpha T^{*} T \sigma_{*}+A^{*}\left(z_{0}-A \sigma_{*}\right) \in C^{0}
$$

and (9) has been proved.

Again from (8) $a-f_{*} \in f_{*}^{\perp}$. It follows, that $\left(a-f_{*}, f_{*}\right)_{F}=0$. But $f_{*}=\left(T \sigma_{*}, A \sigma_{*}\right)$, so

$$
\begin{aligned}
\left(\left[0_{Y}, z_{0}\right]-\left[T \sigma_{*}, A \sigma_{*}\right],\left[T \sigma_{*}, A \sigma_{*}\right]\right) & =\left(\left[-T \sigma_{*}, z_{0}-A \sigma_{*}\right],\left[T \sigma_{*}, A \sigma_{*}\right]\right) \\
& =-\alpha\left\|T \sigma_{*}\right\|^{2}+\left(z_{0}-A \sigma_{*}, A \sigma_{*}\right) \\
& =-\alpha\left\|T \sigma_{*}\right\|^{2}-\left(A \sigma_{*}-z_{0}, A \sigma_{*}-z_{0}\right)-\left(A \sigma_{*}-z_{0}, z_{0}\right) \\
& =-\Phi_{\alpha}\left(\sigma_{*}\right)-\left(A \sigma_{*}-z_{0}, z_{0}\right)=0 .
\end{aligned}
$$


Therefore $\Phi_{\alpha}\left(\sigma_{*}\right)=\left(z_{0}-A \sigma_{*}, z_{0}\right)$. Note that equality in (11) is reached only for the solution $\sigma_{*}$. We obtain then equation (10), and the theorem has been proved.

We shall look for the solution of problem (5) in a proper basis.

Let $k_{1}, k_{2}, \ldots, k_{N}$ be linearly independent elements of $X$, and $A: X \rightarrow Z=Z^{N}$. The action of the operator $A$ may be represented by

$$
A \sigma=\left(\left(k_{1}, \sigma\right),\left(k_{2}, \sigma\right), \ldots,\left(k_{N}, \sigma\right)\right)
$$

Let $K$ be the space of linear combinations of $k_{1}, k_{2}, \ldots, k_{N}$. The dual operator satisfies

$$
A^{*} \lambda=\sum \lambda_{i} k_{i}
$$

The Hilbert space $Y$ may be represented as a direct sum

$$
Y=(T \operatorname{ker} A) \oplus(T \operatorname{ker} A)^{\perp}
$$

For the solution $\sigma_{*} \in X$ there exists $y_{0} \in T \operatorname{ker} A$, with $y_{0}=T x_{0}$ for some element $x_{0} \in \operatorname{ker} A$, and there exists $y \in(T \operatorname{ker} A)^{\perp}$, so that

$$
T \sigma_{*}=y_{0}+y=T x_{0}+y \text {. }
$$

The following equations can be proved easily.

LEMMA 6.

(1) $\operatorname{ker} A=K^{\perp}$.

(2) $\left(T K^{\perp}\right)^{\perp}=T^{*-1}(H)$, where $H=K \cap(\operatorname{ker} T)^{\perp}$.

(3) If $\operatorname{ker} T \cap \operatorname{ker} A=0_{X}$ and $\operatorname{dim} \operatorname{ker} T=q<\infty$, then

$$
\operatorname{dim} H=\operatorname{dim} K-\operatorname{dim}(\operatorname{ker} T)=N-q
$$

(4) $\left(T^{*-1} h\right)(t)=\left(h(x), G_{+}(x-t)\right)_{X}$, where $h \in(\operatorname{ker} T)^{\perp}$.

Here $G_{+}(x-t)$ is the Green's function with $T G_{+}(x-t)=\delta_{t}$, and $\delta_{t}(v)=v(t), v \in X$. An algorithm for finding a basis in $(T \operatorname{ker} A)^{\perp}$ follows if we use Lemma 6 .

(1) A basis for $H=K \cap(\operatorname{ker} T)^{\perp}$ is looked for

$$
h_{i}=\sum_{j=1}^{N} h_{i j} k_{j}, i=1,2, \ldots, N-q .
$$

(2) If $e_{1}, e_{2}, \ldots, e_{q}$ is a basis for $\operatorname{ker} T$, then

$$
0=\left(h_{i}, e_{k}\right)=\sum_{j=1}^{N} h_{i j}\left(k_{j}, e_{k}\right), i=1,2, \ldots, N-q, k=1,2, \ldots, q
$$


(3) $f_{i}=T^{*-1}\left(h_{i}\right), i=1,2, \ldots, N-q$ is a basis for $T^{*-1}(H)=\left(T K^{\perp}\right)^{\perp}$ $=(T \operatorname{ker} A)^{\perp}$.

(4) For every $y \in(T \operatorname{ker} A)^{\perp}$ there exist $\lambda_{1}, \lambda_{2}, \ldots, \lambda_{N-q}$, so that

$$
y=\sum_{i=1}^{N-q} \lambda_{i} f_{i} .
$$

Now from (12) we have the representation

$$
T \sigma_{*}=T x_{0}+\sum \lambda_{i} f_{i}
$$

Let's introduce the matrices

$$
\begin{aligned}
H & =\left(h_{i j}\right)_{i=1, \ldots, N-q}^{j=1, \ldots, N}, \quad F=\left(\left(f_{i}, f_{j}\right)\right)_{i=1, \ldots, N-q}^{j=1, \ldots, N-q} . \\
\lambda & =\left(\lambda_{1}, \lambda_{2}, \ldots, \lambda_{N-q}\right), \quad r=\left(r_{1}, r_{2}, \ldots, r_{N}\right) .
\end{aligned}
$$

LEMMA 7 . For every $y \in(T \operatorname{ker} A)^{\perp}$ there exists $r \in Z^{N}$, so that

$$
T^{*} y=A^{*} r, \quad r=\lambda H \text {. }
$$

Proof: From the above there exist $\lambda_{1}, \lambda_{2}, \ldots, \lambda_{N-q}$, so that $y=\sum \lambda_{i} f_{i}$. Thus

$$
\begin{aligned}
T^{*} y & =T^{*}\left(\sum \lambda_{i} f_{i}\right)=\sum \lambda_{i}\left(T^{*} f_{i}\right)=\sum \lambda_{i} h_{i}=\sum \lambda_{i} \sum h_{i j} k_{j} \\
& =\sum \sum \lambda_{i} h_{i j} k_{j}=\sum r_{j} k_{j}=A^{*} r, r=\left(r_{1}, r_{2}, \ldots, r_{N}\right) .
\end{aligned}
$$

Here

$$
r_{j}=\sum_{i=1}^{N-q} \lambda_{i} h_{i j}
$$

or, using a matrix form,

$$
r=\lambda H .
$$

Let us denote $v=A \sigma_{*}=\left(\left(k_{1}, \sigma_{*}\right), \ldots,\left(k_{N}, \sigma_{*}\right)\right)$. There exists a relation between $\lambda$ and $v$.

LEMMA 8. $\lambda F=v H^{T}$.

Proof: We have in (13) $T \sigma_{*}=T x_{0}+\sum \lambda_{i} f_{i}$. Thus

$$
\left(T \sigma_{*}, f_{j}\right)=\left(T x_{0}, f_{j}\right)+\left(\sum \lambda_{i} f_{i}, f_{j}\right)=\left(x_{0}, T^{*} f_{j}\right)+\sum \lambda_{i}\left(f_{i}, f_{j}\right)
$$

But $\left(x_{0}, T^{*} f_{j}\right)=\left(x_{0}, h_{j}\right)=0$ because of $x_{0} \in \operatorname{ker} A=K^{\perp}, h_{j} \in H \subset K$. On the other hand,

$$
\left(T \sigma_{*}, f_{j}\right)=\left(\sigma_{*}, h_{j}\right)=\left(\sigma_{*}, \sum h_{j l} k_{l}\right)=\sum h_{j l}\left(k_{l}, \sigma_{*}\right)=\sum h_{j l} v_{l}
$$


Comparing the right sides of (17) and (18), it follows

$$
\lambda F=v H^{T} .
$$

LEMMA 9. If $e_{1}, e_{2}, \ldots, e_{q}$ is a basis for $\operatorname{ker} T$ and $B=\left(b_{j, k}\right)_{j=1,2, \ldots, N}^{k=1,2, \ldots,}$, with $b_{j, k}$ $=\left(k_{j}, e_{k}\right)$, then $r B=0$.

Proof: From (2) and (1) of the algorithm above, it follows that

$$
0=\sum \lambda_{i}\left(h_{i}, e_{k}\right)=\sum \lambda_{i}\left(\sum h_{i j} k_{j}, e_{k}\right)=\sum \lambda_{i} h_{i j}\left(k_{j}, e_{k}\right)=\sum r_{j} b_{k j}, k=1,2, \ldots, q .
$$

Therefore $r B=0$.

From the representation (13) of the solution and Lemma 7 it follows that there exist $x_{0} \in \operatorname{ker} A$ and $r \in Z^{N}$, so that

$$
T^{*} T \sigma_{*}=T^{*} T x_{0}+A^{*} r
$$

The conditions (9) and (10) take the form

$$
\begin{gathered}
-\alpha T^{*} T x_{0}+A^{*}\left(-\alpha r+z_{0}-v\right) \in C^{0} \\
\alpha\left\|T x_{0}+\sum \lambda_{i} f_{i}\right\|^{2}=\left(z_{0}-v, v\right)
\end{gathered}
$$

Let us remark

$$
\left\|T x_{0}+\sum \lambda_{i} f_{i}\right\|^{2}=\left\|T x_{0}\right\|^{2}+\sum \sum \lambda_{i}\left(f_{i}, f_{j}\right) \lambda_{j}=\left\|T x_{0}\right\|^{2}+\lambda F \lambda^{T}
$$

The following theorem is a consequence of Lemmas 7,8 and 9 .

THEOREM 3. The solution $\sigma_{*}$ of the problem (5) may be represented in the form

$$
T \sigma_{*}=T x_{0}+\sum \lambda_{i} f_{i}, x_{0} \in \operatorname{ker} A,
$$

if and only if $x_{0}$ and $\lambda$ satisfy

$$
\begin{gathered}
-\alpha T^{*} T x_{0}+A^{*}\left(-\alpha r+z_{0}-v\right) \in C^{0} \\
\alpha\left(\left\|T x_{0}\right\|^{2}+\lambda F \lambda^{T}\right)=\left(z_{0}-v, v\right),
\end{gathered}
$$

where $r \in Z^{N}, v \in Z^{N}$ are related to $\lambda$ by

$$
r=\lambda H, \quad \lambda F=v H^{T}, \quad r B=0 .
$$

Let us consider problem (5) in the following situation. The knots

$$
a=t_{1}<t_{2}<\ldots<t_{N}=b
$$


and values $z_{1}, z_{2}, \ldots, z_{N}$ are given in the interval $[a, b]$. Let $X=W_{2}^{n}[a, b]$ be the Sobolev space of functions with the usual norm

$$
\|f\|_{W_{2}^{n}}^{2}=\sum_{j=0}^{n}\left\|f^{(j)}\right\|_{L^{2}[a, b]}^{2}
$$

Let $Y=L_{2}[a, b]$ be the space of square integrable functions. Let $T=\frac{d^{n}}{d t^{n}}$ and let $A: W_{2}^{n} \rightarrow Z=Z^{N}$ be the operator-trace of the function,

$$
A u=\left(u\left(t_{1}\right), u\left(t_{2}\right), \ldots, u\left(t_{N}\right)\right)
$$

Let

$$
C=\left\{\sigma \in W_{2}^{m}[a, b]: \frac{d^{m} \sigma}{d t^{m}} \geqslant 0\right\}
$$

the subset of $m$ - convex functions in $X$, where $m \leqslant n$.

LEMMA 10.

(1) $A$ and $T$ are linear bounded operators.

(2) $C$ is a closed convex subset of $X$.

(3) If the number of the knots $N$ is greater or equal to the order of the differentiation $n$, then

$$
\operatorname{ker}\left\{\frac{d^{n}}{d t^{n}}\right\} \cap \operatorname{ker} A=0_{X},
$$

and $\operatorname{ker} T+\operatorname{ker} A$ is closed in $X$.

PROOF: To prove (3) note ker $\frac{d^{n}}{d t^{n}}=\left\{x: \frac{d^{n} x}{d t^{n}}=0\right\}$ consists of polynomials of order smaller then $n$; while $\operatorname{ker} A=\left\{u: u\left(t_{i}\right)=0, i=1,2, \ldots, N\right\}$ contains functions with zeros at these $N$ points. The intersection of these kernels is empty, because a polynomial of degree smaller than $n$ cannot have $N>n-1$ zeros.

Obviously $\operatorname{ker} T$ and $\operatorname{ker} A$ are closed, their sum is also closed.

Lemma 10 and Theorem 3 give the following result.

THEOREM 4. The problem

$$
\phi_{\alpha}\left(\sigma_{*}\right)=\min _{\sigma \in C}:\left\{\phi(\sigma)=\alpha\left\|\frac{d^{n} \sigma}{d t^{n}}\right\|_{L_{2}}^{2}+\sum_{i=1}^{N}\left(\sigma\left(t_{i}\right)-z_{i}\right)^{2}\right\}
$$

has unique solution $\sigma_{*}$ for $N \geqslant n$. When $\sigma^{(m)} \geqslant 0$,

$$
\sum_{i=1}^{N}\left(z_{i}-\sigma_{*}\left(t_{i}\right)\right) \sigma\left(t_{i}\right) \leqslant \alpha \int \sigma^{(n)}(t) \sigma_{*}^{(n)}(t) d t
$$

with equality only for the solution $\sigma_{*}(t)$. 
Proof: The problem has unique solution by the previous results. The condition (9) in this case is

$$
-\alpha\left(\frac{d^{n}}{d t^{n}}\right)^{*}\left(\frac{d^{n} \sigma_{*}}{d t^{n}}\right)+\sum_{i=1}^{n}\left(z_{i}-\dot{\sigma}_{*}\left(t_{i}\right)\right) k_{i} \in C^{0}
$$

From (11) it follows, that for all $\sigma$ with $\sigma^{(m)} \geqslant 0$ it must be performed

$$
\sum_{i=1}^{N}\left(z_{i}-\sigma_{*}\left(t_{i}\right)\right) \sigma\left(t_{i}\right) \leqslant \alpha \int \frac{d^{n} \sigma_{*}}{d t^{n}} \frac{d^{n} \sigma}{d t^{n}} d t
$$

Equality is acheived only for the solution $\sigma=\sigma_{*}$

$$
\alpha\left\|\sigma_{*}^{(n)}\right\|_{L^{2}}^{2}=\sum\left(z_{i}-\sigma_{*}\left(t_{i}\right)\right) \sigma_{*}\left(t_{i}\right)
$$

In fact this equality is equivalent to the condition (10).

For $T=\frac{d^{n}}{d t^{n}}$ it is known, that

$$
G_{+}(x-t)=\frac{(x-t)_{+}^{n-1}}{(n-1) !}
$$

A basis for $\operatorname{ker} T$ is $\left\{1, t, t^{2}, \ldots, t^{n-1}\right\}$, and therefore $\operatorname{dim} \operatorname{ker} T=q=n$. Then

$$
h_{i}=\sum_{j=1}^{N} h_{i j} k_{j}
$$

so that $\left(h_{i}, t^{k}\right)=0, i=1,2, \ldots, N-n, k=0,1, \ldots, n-1$. It follows; that

$$
\sum_{j=1}^{N} h_{i j} t_{j}^{k}=0, i=1,2, \ldots, N-n, k=0,1, \ldots, n-1 .
$$

We have

$$
f_{i}(t)=\left(\sum_{j=1}^{N} h_{i j} k_{j}(x), \frac{(x-t)_{+}^{n-1}}{(n-1) !}\right)=\sum_{j=1}^{N} h_{i j} \frac{\left(t_{j}-t\right)_{+}^{n-1}}{(n-1) !}, i=1,2, \ldots, N-n .
$$

By Theorem 3 the solution of (20) has the representation

$$
\sigma_{*}^{(n)}(t)=x_{0}^{(n)}+\sum \lambda_{i} \sum \frac{h_{i j}\left(t_{j}-t\right)_{+}^{n-1}}{(n-1) !}=x_{0}^{(n)}+\sum r_{j} \frac{\left(t_{j}-t\right)_{+}^{n-1}}{(n-1) !}
$$

where the condition $r B=0$ is equivalent to

$$
\sum_{j=1}^{N} r_{j} t_{j}^{k-1}=0, k=1,2, \ldots, n
$$


On integrating $n$ times,

$$
\sigma_{*}(t)=x_{0}(t)+\sum_{j=1}^{N} r_{j} \frac{\left(t_{j}-t\right)_{+}^{2 n-1}}{(2 n-1) !}+\sum_{k=0}^{n-1} c_{k} t^{k} .
$$

The function

$$
s(t)=\sum_{j=1}^{N} r_{j} \frac{\left(t_{j}-t\right)_{+}^{2 n-1}}{(2 n-1) !}+\sum_{k=0}^{n-1} c_{k} t^{k}
$$

under the constraints (21) is a natural spline ([1]) of degree $2 n-1$ with knots $t_{1}, t_{2}, \ldots, t_{N}$. Since the restriction of $s(t)$ over $\left(-\infty, a=t_{1}\right)$ and $\left(t_{N}=b, \infty\right)$ is the polynomial $\sum c_{k} t^{k}$ of degree $n-1$, we have the following result.

THEOREM 5. The solution of the problem (20) is a sum of a function $x_{0}(t)$ with zero-crossings $t_{1}, t_{2}, \ldots, t_{N}$ and a natural spline $s(t)$ of degree $2 n-1$ with knots in these points.

\section{REFERENCES}

[1] B.D. Bojanov, H.A. Hakopian and A.A. Sahakian, Spline functions and multivariate interpolations, Mathemtics and its Applications 248 (Kluwer Academic Publishers, Dordrecht, 1993).

[2] S. le Cessie and E.J. Balder, 'A note on monotone interpolation and smoothing splines', Numer. Funct. Anal. Optim. 15 (1994), 47-54.

[3] C.K. Chui, F. Deutsch and J. Ward, 'Constrained best approximation in Hilbert space', Constr. Approx. 6 (1990), 35-64.

[4] C.K. Chui, F. Deutsch and J. Ward, 'Constrained best approximation in Hilbert space II', J. Approx. Theory 71 (1992), 213-238.

[5] P.-J. Laurent, Approximation et optimisation (Hermann, Paris, 1972).

Department of Descriptive Geometry

University of Architecture,

Civil Engineering and Geodesy

Boul. Hr. Smirnensky 1

Sofia 1421

Bulgaria

e-mail: dichevan_fgs@bgace5.uacg.acad.bg 